\title{
"Pulse Oximetryin Pulp Vitality Test - Comparative Evaluation with the Conventional Techniques
}

\author{
Dr. Prasanth Balan M.D.S ${ }^{1,}$ Dr Varghese N.O M.D.S ${ }^{2,}$ \\ Dr Jolly Mary Varughese M.D. $S^{3}$, Dr. Nileena R kumar M.D.S $S^{4}$ \\ Dr Priya R MDS ${ }^{5}$ \\ ${ }^{1}$ Reader, Dept of Conservative Dentistry and Endodontics, Govt Dental College Calicut. \\ ${ }^{2}$ Principal \& Professor PMS Dental College, Vattapara \\ ${ }^{3}$ Principal\& Professor Govt Dental College, Trichur \\ ${ }^{4}$ Associate professor Department Of Oral Medicine Govt Dental College Calicut \\ ${ }^{5}$ Reader Dept Of Conservative Dentistry \& Endodontics ,Amrita School Of Dentistry, Kochi
}

\begin{abstract}
Context:This studycompares the clinical effectiveness of newly designed pulse oximetry probe over conventional technique of electric pulp testing and thermal testing.

Aims:To evaluate the clinical efficacy of customized pulseoximeter probe over the conventional thermal and electrical testing of tooth vitality

Settings and Design:The conventional pulse oximetry probe was customized for the purpose of pulp vitality test and was used in this study and registered excellent readings.

Methods and Material: One hundred and twenty five patients in the age group 15-35 years, were included in this study. Out of them, fifty patients with normal maxillary central incisors served as group I, and seventy-five patients in whom one of the maxillary incisors was traumatized served as group II.As a control population to confirm the readings, 10 known non-vital maxillary central incisors with complete endodontic fillings were tested.

Statistical analysis used: Spearman correlation test \& Mann- Whiteny U test.

Results:In all the groups, The 10 known non-vital teeth with complete endodontic fillings recorded oxygen saturation $\left(\mathrm{SaO}_{2}\right)$ values of $0 \%$ and showed no response to electrical and thermal testing procedures.

In group IThe $\mathrm{SaO}_{2}$ values obtained on the teeth were correlated with the electric pulp tester readings. The correlation between the $\mathrm{SaO}_{2}$ readings and electrical testing readings was found to be negative. The correlation was highly significant $(p<0.001)$. The correlation of the pulse rate readings from the pulp with electric pulp tester readings were also found to be significant $(p<0.05)$.

In group II Oxygen saturation and pulse rate value of the tooth showed a negative correlation with electric pulp tester.

Conclusions: Present study shows that pulseoximetrywith probe modification can be successfully used for pulp vitality test.

Key-words: Pulp vitality, pulse oximetry, Key Messages: This study indicates that pulse oximetry with adequate modification in the probe can be successfully used for the purpose for pulp vitality test by measuring the oxygen saturation of dental pulp.
\end{abstract}

\section{Introduction}

One of the crucial diagnostic procedures in the field of endodontics is the assessment of pulpal vitality. Since the pulp tissue cannot be directly inspected, we have to use indirect methods to test the pulpal vitality. The most commonly used tests in endodontic practice are thermal tests and electrical tests. Thermal test works on the principle of flow of dentinal fluid at temperature changes, which lead to the movement of the odontoblastic process and subsequent mechanical stimulation of the pulpal nerves. On the other hand, in electrical tests an electric current is conducted through the tooth giving an electrical stimulation to the pulpal nerves. These modalities fall short of the ideal pulp tests on several criteria. All these tests are subjective tests that depend upon the patient's perceived response to a stimulus as well as the dentist's interpretation of that response. Moreover, these testing methods have the potential to produce an unpleasant and occasionally painful sensation. Inaccurate results may often result, for example, in thermal test when ice melts and drips onto adjacent teeth or gingival tissues ${ }^{[1]}$, or when electric current applied to the tooth surface is conducted to the periodontal ligament ${ }^{[2]}$, thus stimulating periodontal nerve fibres. False negative responses may also occur in cases of calcific metamorphosis, in teeth with immature root formation, or subsequent to an impact injury. 
Patients' erratic response to avoid a painful stimulus has also been demonstrated in an analysis of electric pulp testers.

Another problem with the present pulp testing methods is that they only indirectly monitor pulp vitality by measuring the neural responses and not the blood circulation. Since pulp vitality is purely a function of healthy vasculature ${ }^{[3,4,5]}$ a vital pulp with an intact vasculature may test non vital if only its neural component is injured. This situation is commonly encountered with recently traumatized teeth. ${ }^{[6]}$ On the other hand, the pulp nerve fibers are more resistant to necrosis than the vasculature tissues; and thermal or electric testing of the pulpal neural response may also result in false positive results if only the pulp vasculature is damaged ${ }^{[7]}$.

For electric and thermal testing to be effective, the pulp must have a sufficient number of mature neurons. However, both the primary and young permanent teeth are not fully innervated with alpha myelinated neurons, the neural components that are responsible for the pulpal response. Permanent teeth may not exhibit full alpha - myelinated axon innervations until 4-5 years after eruption. These reduced numbers of pain receptors in these teeth make them less responsive to stimuli and therefore more susceptible to give negative results with thermal and electrical testing ${ }^{[8]}$.Considering all these limitations, present pulp testing with thermal and electrical methods cannot be cited as reliable pulp vitality tests. This emphasizes the need for a technique which directly assesses the pulpal circulation as a measure of pulp vitality.

Pulse oximetry is a completely objective test, requiring no subjective responses from the patients. It directly measures the blood oxygen saturation levels. It was proven effective and is routinely used in medical applications with finger, toe, foot and ear probes.

The present study was undertaken to detect the vascularity of the pulp as a measure of tooth vitality in normal and traumatized teeth.

Aim of the present study was

(i) To evaluate the clinical efficacy of pulse oximeter over the conventional thermal and electrical testing of tooth vitality.

The objective of using the pulse oximeter is to assess the blood circulation of the pulp by:-

1) Evaluation of the oxygen saturation of the pulp

\section{Evaluation of the pulse rate readings from the pulp vasculature}

\section{(1) Pulse Oximeter Machine And Probe Design}

\section{Subjects and Methods}

Aster 2 pulse oximeter instrument was used in this study (photograph 1). Initially, a pulse oximeter with a pediatric probe (as used imedical\& surgical specialty) was used which facilitated manual placement on the tooth surface.

It was found that the probe could not be held in close adaptation to the tooth surface and retained in that position without movement for sufficient length of time, so it became necessary to develop a customized probe to suit the nature of the study.

An extracted maxillary central incisor was taken to the lathe to provide a template for designing the probe. The probe was made of bakelite and the light source and sensor from the pediatricprobe(Photograph II ) was incorporated into this newly designed probe. This probe would closely adapt to the tooth surface. However, even this probe was unable to produce an acceptable reading.

The probable causes of the failure at this step were assessed and could be due to

1) low intensity of the incident light to the tooth surface

2) low penetration of the light through calcified tissue of the tooth or due to high absorption of the light by calcified tissue of tooth

3) low intensity of the signals reaching in the sensor

4) scattering of the incident light from the tooth surface

(1)The intensity of the incident light was the property of the equipment used and hence could not be easily altered. Any attempt to alter the intensity of light or to change the source to laser light would require all the design and electronics of the equipment to be changed. Therefore, it was not practical.

(2)In order to know the transmission and absorption of light through the enamel, an absorption spectrometry of the enamel was carried out. It was studied by subjecting a layer of powdered enamel and a thin section of the tooth to absorption spectrometry. It was seen that the specimen exhibited a flat spectrometry between $200 \mathrm{~nm}$ and $800 \mathrm{~nm}$ (Fig $1 \&$ Fig II). This means that light within this range of 
wavelength is transmitted entirely, without any absorption by the calcified tissue. The wavelength of light source used in pulse oximeter comes in this range and so it should pass through the tooth.

(3)Next attempt was made to prevent the scattering of the incident light. This was achieved by placing a Plano convex lens in the path of the incident light in order to converge the light beam. An $8 \mathrm{~mm}$ diameter lens with a focal length of $10 \mathrm{~mm}$, was found to be satisfactory.

The probe thus customized(photograph III) was used in this study and registered excellent readings. (photograph IV)

\section{2) Patient selection}

One hundred and twenty five patients in the age group 15-35 years were selected out of them, fifty patients with normal maxillary central incisors served as group I, and seventy-five patients in whom one of the maxillary incisors was traumatized served as group II. In both groups, two maxillary central incisors were subjected to vitality test by thermal, electrical means and by pulse oximetry.

Pulse oximeter readings from the patient's index finger served as the control sample for the comparison of the pulp oxygen saturation values with the patient's systemic oxygen saturation values.

The readings obtained from the pulse oximeter were correlated to conventional electrical pulp vitality tester (Parkell Pulp Vitality Tester) and thermal testing using ice stick.

The patient's oxygen saturation $\left(\mathrm{SaO}_{2}\right)$ values were first measured on the index finger for the comparison of the pulp oxygen saturation values with the patient's systemic oxygen saturation values. $\mathrm{The}^{\mathrm{SaO}} \mathrm{S}_{2}$ values of the teeth were recorded by placing the probe on cervical 3rd of the crown so that the light would travel from facial to lingual aspect through the middle of the crown( Photograph IV ). The values were recorded after 30 seconds of monitoring each tooth.

The vitality of these teeth was subsequently recorded by electric pulp tester and ice stick . The readings were tabulated and statistically analyzed. Spearman correlation test was the statistical test applied in order to find out the correlation between electric pulp tester and pulse oximetry readings. Thermal test findings and pulse oximetry readings in the traumatized teeth were correlated using Mann- Whiteny $\mathrm{U}$ test.

\section{Results}

In all the groups, The 10 known non-vital teeth with complete endodontic fillings recorded oxygen saturation $\left(\mathrm{SaO}_{2}\right)$ values of $0 \%$ and showed no response to electrical and thermal testing procedures.

In group I, the mean $\mathrm{SaO} 2$ values measured on the patient's finger was $98.90 \%$ (S. D. $= \pm 1.6817$ ) and the pulse rate from the finger averaged $81.07 \%$ (S. D. $= \pm 7.2548$ ). The $\mathrm{SaO}_{2}$ values on permanent central incisors averaged $95.20 \%$ ( S.D. $= \pm 2.1130)$ and the pulse rate readings from the pulp averaged $61.59 \%$ ( $\mathrm{S} . \mathrm{D}= \pm 6.4245)$. The electric pulp tester reading of group I averaged $3.03 \%$ (S.D. \pm 0.5383 ).

The $\mathrm{SaO}_{2}$ values obtained on the teeth were correlated with the electric pulp tester readings. The correlation between the $\mathrm{SaO}_{2}$ readings and electrical testing readings was found to be negative i.e. as the values of the electrical pulp testing reading increased, the $\mathrm{Sa}_{2}$ values decreased. The correlation was highly significant $(\mathrm{p}<0.001)$.

The correlation of the pulse rate readings from the pulp with electric pulp tester reading was found to be significant $(\mathrm{p}<0.05)$.

Out of the seventy five traumatized teeth in group II, twenty three teeth were non-vital and showed no pulse oximeter readings, no electrical pulp test readings and negative readings for thermal test.

The rest of the teeth were vital and showed $\mathrm{SaO}_{2}$ values which averaged $49.63 \%$ (S.D. $= \pm 34.0872$ ) Pulse rate reading from the teeth averaged $32.81($ S.D. $= \pm 22.4858)$. Oxygen saturation of the tooth showed a negative correlation with electric pulp tester (Table I). Pulse rate value from the tooth also showed a negative correlation with electric pulp tester ( $\mathrm{p}$ value $<0.05$ )( Table II) .Oxygen saturation and pulse rate values from the tooth were statistically analyzed with thermal test readings using Mann-Whitney $U$ test which is a nonparametric test. Mann-Whitney $U=166.000$, pvalue $=0.000$ ) the result were found to be highly significant $(\mathrm{p}<0.001)$.

\section{Discussion}

The vitality of the pulp-dentin complex is fundamental to the functional life of the tooth and is a priority for targeting clinical management strategies ${ }^{[9]}$. Pulp vitality test is crucial in monitoring the state of health of dental pulp, especially after traumatic injuries ${ }^{[10]}$. Pulp vitality is determined by blood circulation, i.e. the health of the vasculature of the dental pulp. Thus, vitality tests should determine whether the circulation is intact in the dental pulp.

Two wavelengths of light are used; 660 nanometers (red) and 940 nanometers (near infrared). At 660 $\mathrm{nm}$, reduced hemoglobin absorbs about ten times as much light as oxyhemoglobin. At the infrared wavelength $(940 \mathrm{~nm})$, the absorption coefficient of oxyhemoglobin is greater than that of reduced hemoglobin. The pulse 
oximeter directly senses the absorption of red and infrared light; and the ratio of pulsatile to nonpulsatile light at the red and infrared wavelengths are translated through complex signal processing to a function of the arterial oxygen saturation $^{[11]}$. A microprocessor integrates the data, and through an elaborate calibration algorithm based on human volunteer data, the oxygen saturation can be estimated.

Very few studies have been reported in the literature regarding the application of pulse oximetry in the vitality assessment of the teeth.Earlier studies by Schnettler and Wallace ${ }^{[12]}$ reported a correlation between pulp and systemic oxygen saturation readings using a modified ear pulse oximeter probe on a tooth. A K Munshiet al (2002) conducted a study on pulse oximetry, to evaluate its reliability as a diagnostic instrument in pulpal vitality testing $^{[8]}$. Siddeswaran et al (2011) conducted a study to evaluate the efficacy of pulse oximetry as pulp vitality test ${ }^{[13]}$.Gopikrishna et al. ${ }^{[14]}$ developed a custom-made pulse oximetersensor holder for an existing NellcorOxiMax Dura-Y D-YS multisite oxygen sensor (Tyco Healthcare Group LP, Pleasanton, CA) and showed the utility of the pulse oximeter dental probe in assessment of human pulp vitality. V Gopikrishna et al evaluated the efficacy of pulse oximetry in pulp vitality in recently traumatized teeth ${ }^{[14][15][16]}$ and later he patented the pulse oximetry probe for dental use. The result of the present study also confirmed the efficacy of a customized pulse oximetry probe for the use of pulp vitality test.

\section{Conclusion}

Pulp vitality has been an elusive entity to the oral diagnostician and attempts at its accurate assessment have always fallen short of the goal. The current trend is towards mapping the blood flow of the pulp rather than depending on indirect measures of the integrity of neural elements. Pulse oximetry has been over looked in this regard and its potential as an adept diagnostic tool untapped to date .The present study was aimed at evaluating the reliability and accuracy of the pulse oximeter as a pulp vitality tester and assessing its superiority over conventional electrical and thermal methods by clinical trials.

\section{References}

[1]. Walton RE, Torabibinijad, M. B., Saunders,W,B. "Principles and practice of Endodontics Philadelphia"(1989),61 62 .

[2]. Rowe, A.H.R., Piff., Ford., T. R. "The assessment of pulp vitality" Int. Endod J, (1990); 23 : $77-83$.

[3]. Craig,W.Noblett, Lisa, R. Wilcon, et al " Detection of pulpalcirculation in vitro by pulse oximetry" Journal of Endodontics(1996),22,1.

[4]. Noblett, W.C., Wilcox, L.R., Scammon, F. Johnson, W.T., "Detection of pulpal circulation in vitro by pulse oximetry". J. Endod. (1996); 22:1-5.

[5]. Sasona T, Onodera D, Hashimoto K. " Possible application of transmitted laser light for the assessment of human pulp vitality part:2 Increased Laser power for enhanced detection of pulpal blood flow" Dent Traumatol (2005) 21:37-41.

[6]. Curt,Goho. Pulse oximetry evaluation of vitality in primary immature permanent teeth. Pediatric dentistry (1999);21 ( 2): :125-127.

[7]. Ikeda H, Sudha H, "subjective sensation and objective neural discharge recorded from clinically nonvital and intact teeth" J endod(1998) 24:552-556.

[8]. Munshi AK, Hegde AM, Radhakrishnan S. Pulse OximetryA diagnostic instrument in pulp vitality testing. J Pediatric Dent Winter 2001;26(2):141-45.

[9]. Anthony, J, Smith;Vitality of the Dentin-Pulp Complex in Health and Disease, Growth Factors as Key Mediators Journalof Dental Education (2003) 67;6:678-688.

[10]. Samraj RV, Indira R, Srinivasan MR, Kumar A. Recent Advances in pulp vitality testing. Endodontol 2003;15:14-19

[11]. Salyer JW. Neonatal and pediatric pulse oximetry. Respir care 2003; 48:386-97.

[12]. Schnettler JM, Wallace JA. Pulse oximeter as a diagnostic tool of pulp vitality. J Endod1991;17:488 -90.

[13]. SiddheswaranV,RohitA,Vasundhara S. Pulse oximetry A diagnostic instrument in pulpal vitality testing - An in vivo study. W.J of Dentisrty 2011;2(3):225-230.

[14]. Gopikrishna V, Kandaswamy D, Tina Gupta K, Assessment of the efficacy of an indigeniously developed pulse oximeter dental sensor holder for pulp vitality testing. Ind J Dent Res 2006; 17(3):111-113

[15]. Gopikrishna V, Kush T, Deivanayagam K. Comparison of electrical, thermal and pulse oximetry methods of assessing pulp vitality in traumatized teeth. J Endod 2007; 33:531-535.

[16]. Gopikrishna V, Kandaswamy D, Tinagupta K. Evaluation of efficacy of a new custommade pulse oximeter dental probe in comparison with the electrical and thermal tests for assessing pulp vitality. J Endod 2007;33:411-4 


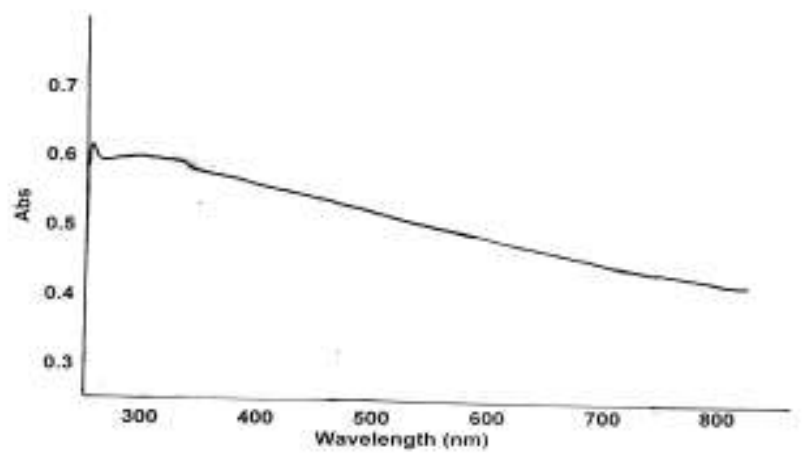

Fig 1:Absorption Sperctrometric Analysis Report Of Enamel Powder

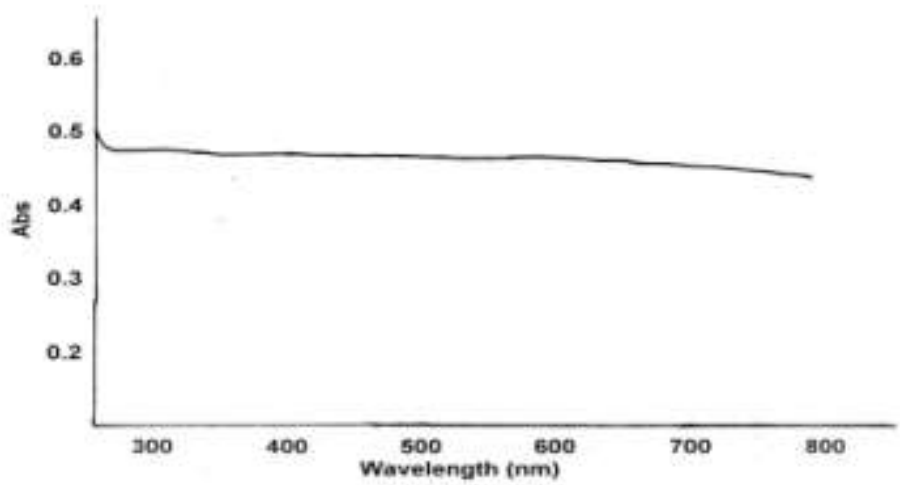

Fig 2:Absorption Sperctrometric Analysis Report Of Enamel Powder

Table I: Showing the correlation between the Electric pulp tester andOxygen saturation in Group II

\begin{tabular}{|c|c|c|c|}
\hline Group I & & EPT & O2TOOTH \\
\hline $\begin{array}{c}\text { Spearman's } \\
\text { correlation } \\
\text { EPT }\end{array}$ & Correlation Coefficient & 1.000 & -.361 \\
\cline { 2 - 4 } & Sig.(2-tailed) &. & .001 \\
\hline $\begin{array}{c}\text { Spearman's } \\
\text { correlation } \\
\text { O2TOOTH }\end{array}$ & Correlation Coefficient & -.361 & 1.000 \\
\cline { 2 - 4 } & Sig. (2-tailed) & .001 &. \\
\hline
\end{tabular}

pvalue $<0.05$ (significant correlation between EPT\& O2TOOTH)

Table II: Showing the correlation between the electric pulp tester andPulse rate readingsin Group II

\begin{tabular}{|c|c|c|c|}
\hline Group I & & EPT & $\begin{array}{c}\text { PR } \\
\text { TOOTH }\end{array}$ \\
\hline $\begin{array}{c}\text { Spearman's } \\
\text { correlation } \\
\text { EPT }\end{array}$ & Correlation Coefficient & 1.000 & -.570 \\
\cline { 2 - 4 } & Sig.(2-tailed) & $\cdot$ & .000 \\
\hline
\end{tabular}




\begin{tabular}{|c|c|c|c|}
\hline $\begin{array}{l}\text { Spearman's } \\
\text { correlation } \\
\text { PRTOOTH }\end{array}$ & Correlation Coefficient & -.570 & 1.000 \\
\cline { 2 - 4 } & Sig. (2-tailed) & .000 &. \\
\hline
\end{tabular}

pvalue $<0.05$ (significant correlation between EPT\& PRTOOTH)

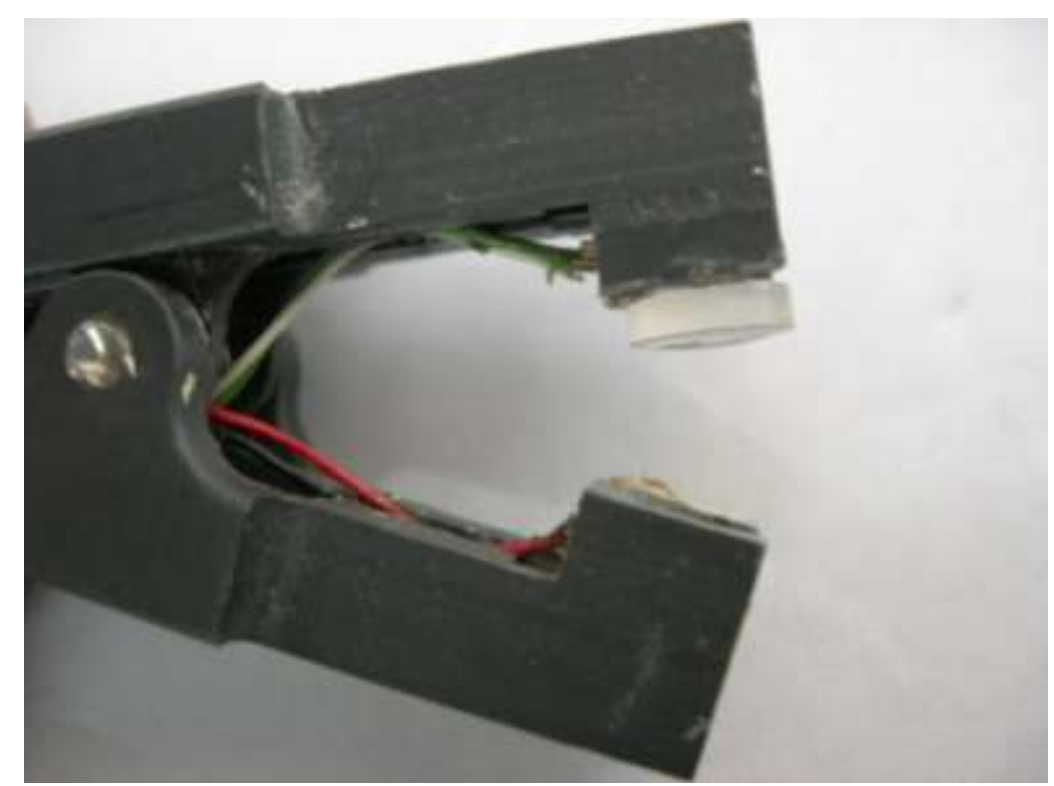

Photo III

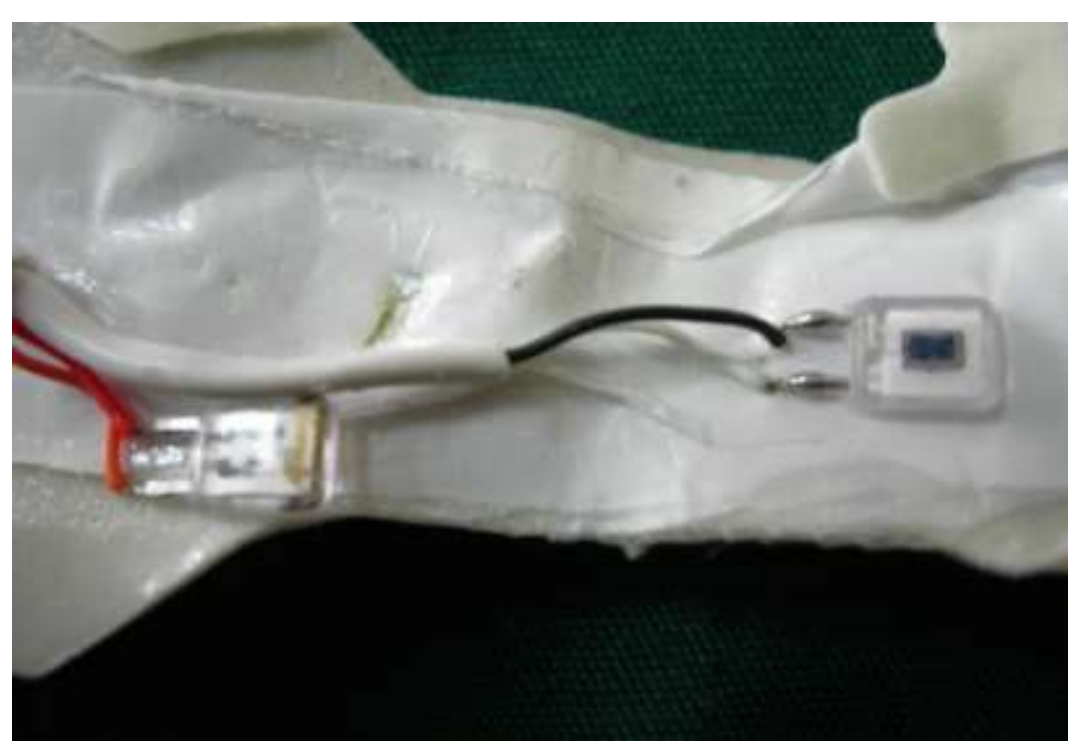




\section{PHOTO II}

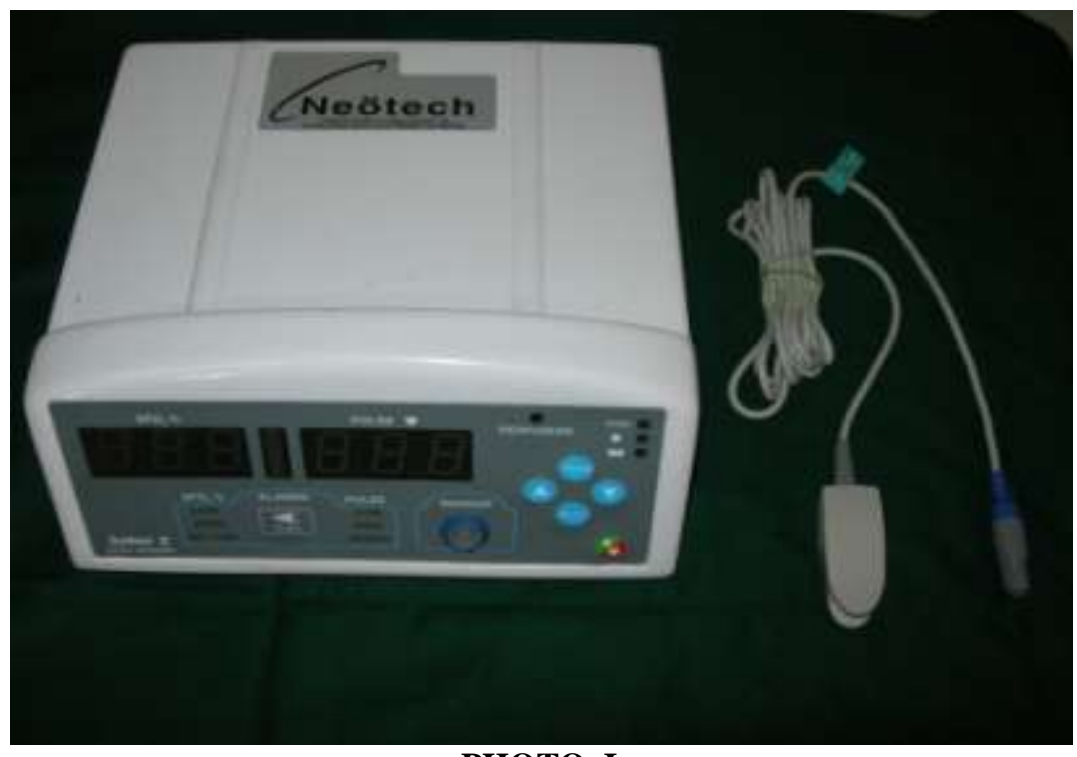

PHOTO I

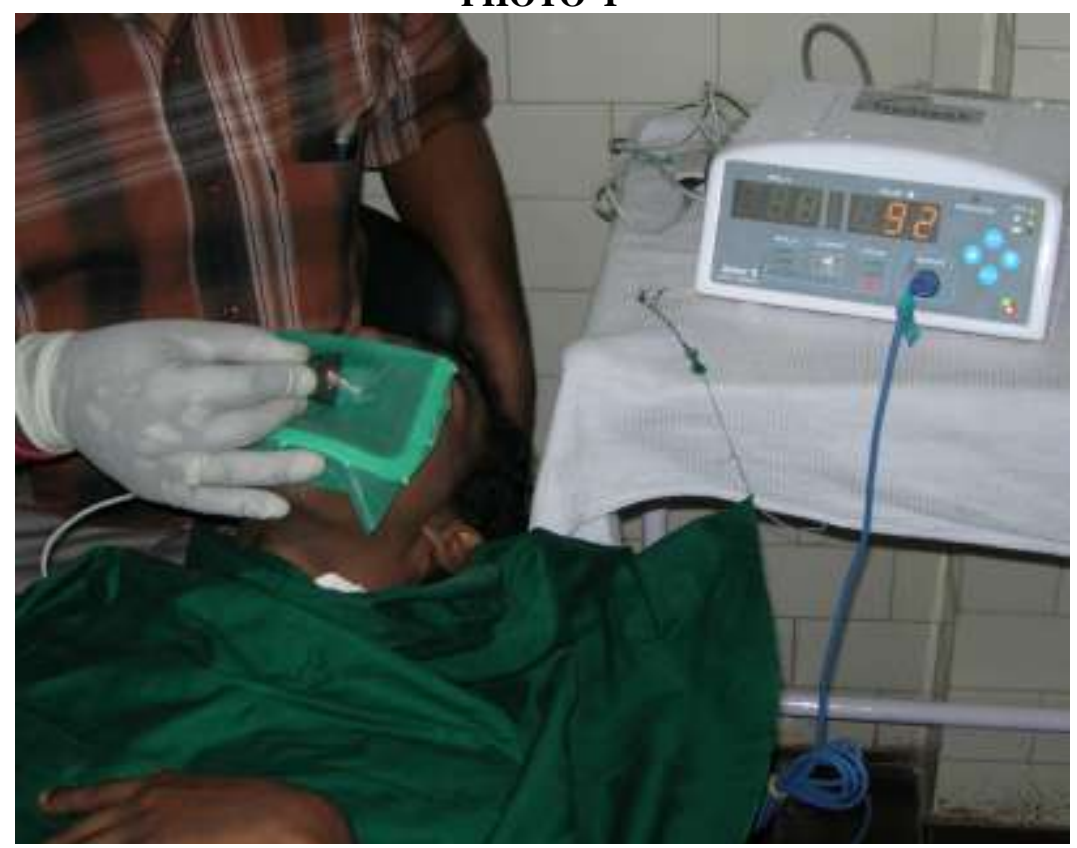

Photo IV 